Herjan van der Steeg

Jesse P. van Akkeren

Saskia Houterman

Rudi M. H. Roumen

\section{Validation of the urine column measurement as an estimation of the intra-abdominal pressure}

Received: 13 May 2008

Accepted: 6 December 2008

Published online: 6 January 2009

(C) The Author(s) 2008. This article is

published with open access at

Springerlink.com

H. van der Steeg (『)

Department of Surgery, University Medical

Centre St. Radboud, Nijmegen,

The Netherlands

e-mail: h.vandersteeg@chir.umcn.nl

Tel.: +31-24-3611111

Fax: +31-24-3540501

J. P. van Akkeren

Department of Intensive Care,

Máxima Medical Centre, Veldhoven,

The Netherlands

S. Houterman

MMC Academy, Máxima Medical Centre,

Veldhoven, The Netherlands

R. M. H. Roumen

Department of Surgery, Máxima Medical

Centre, Veldhoven, The Netherlands
Abstract Objective: To evaluate the efficacy of the urine column (UC) measurement compared to the intravesicular pressure (IVP) measurement as an estimation of intraabdominal pressure (IAP) in patients with IAP up to $30 \mathrm{mmHg}$. Methods: Fifteen patients undergoing a laparoscopic cholecystectomy were studied. All patients were catheterized. IVP measurements were performed using a pressure transducer connected to the culture aspiration port. UC measurements were done by holding up the tubing against a measuring rod. The symphysis pubis was used as the zero-reference. IAP was raised from 0 to $30 \mathrm{mmHg}$ using increments of $5 \mathrm{mmHg}$, during which first the IVP and then UC measurement series were recorded endexpiratory. Fifty and $100 \mathrm{ml}$ of saline were used as a priming volume.

Results: The IVP and UC measurements showed a significant correlation with IAP. Comparing IVP and UC showed a correlation of 0.91 $(p<0.001)$ for $50 \mathrm{ml}$ and 0.87 $(p<0.001)$ for $100 \mathrm{ml}$ of saline as a priming volume. Using $50 \mathrm{ml}$ of saline, UC was $0.68 \mathrm{mmHg}$ higher than IVP $(95 \% \mathrm{CI}-7.21$ to

$+5.85 \mathrm{mmHg}$ ). For $100 \mathrm{ml}$ of saline, UC was $1.23 \mathrm{mmHg}$ lower than IVP (95\% CI -7.41 to $+9.87 \mathrm{mmHg}$ ). Conclusion: UC measurement shows significant correlation to IVP measurement as an estimation of the IAP. Further study needs to be done to conclude whether UC measurement is a reliable clinical alternative to IVP measurement.

Keywords Gastrointestinal problems - Peri-operative care . Multiple organ failure

\section{Introduction}

In recent literature, intra-abdominal hypertension (IAH) and the abdominal compartment syndrome (ACS) have encountered increasing attention [1-3]. The lack of uniformity in definitions for these two entities makes comparisons between different studies difficult $[4,5]$, and has led to a set of proposed definitions [6]. IAH is defined by a sustained or repeated pathological elevation in intraabdominal pressure (IAP) $\geq 12 \mathrm{mmHg}$. The ACS is a sustained IAP $>20 \mathrm{mmHg}$ (with or without an abdominal perfusion pressure $<60 \mathrm{mmHg}$ ) that is associated with new organ dysfunction or failure [6].

Indirect methods to obtain the most reliable estimation of the IAP are intra-gastric, inferior vena cava, or intravesicular pressure (IVP) measurements [7]. IVP measurement has become the gold standard in contemporary medical literature. Kron et al. [8] were the first to describe this method, later on revised by others $[9,10]$.

IVP measurement entails using a pressure transducer, prone to mechanical failure and it is somewhat laborious in performing. An alternative method to estimate the IAP 
is the so-called urine column (UC) measurement or Utube technique, first described by Harrahill [11]. This easy and straightforward method entails raising the urine catheter and connected tubing, hereby measuring the level of the UC to the symphysis pubis, which is used as a zeroreference.

The main aim of the current study was to evaluate the efficacy of the UC measurement compared to the IVP measurement as an estimation of IAP in patients with IAP up to $30 \mathrm{mmHg}$. In addition, we undertook this study to determine the most reliable bladder volume as a priming volume.

The results of this study have been presented at the Third World Congress on the Abdominal Compartment Syndrome in Antwerp, March 2007 and published as an abstract [12].

\section{Methods}

Study population

Fifteen patients undergoing a laparoscopic cholecystectomy between May 2005 and January 2006 in the Máxima Medical Centre in Veldhoven, the Netherlands, were included in this prospective, non-randomized study. Exclusion criteria were ventricular dysfunction $(\mathrm{EF}<25 \%)$, COPD $\left(\mathrm{PaCO}_{2}>50 \mathrm{mmHg}, \mathrm{FEV} 1<1 \mathrm{~L}\right)$, previous bladder operations, neurogenic bladder dysfunction, recurrent urinary tract infections, pregnancy and patients under the age of 18 . For each patient Body Mass Index $\left[\right.$ BMI $\left.\left(\mathrm{kg} / \mathrm{m}^{2}\right)\right]$ was recorded. The study was approved by the local ethics committee. All patients gave written informed consent.

\section{IVP and UC measurements}

All procedures were performed in the operating theatre under general anesthesia. Patients were mechanically ventilated and catheterized with a Foley catheter. A urinary drainage bag (Urimeter, Unomedical, Birkerød, Denmark) was attached. A urine column-measuring rod, developed for this study by the hospital technical department, was placed between the patient's legs. The catheter and proximal tubing were attached to the horizontally placed bar of the rod, using the symphysis pubis as zeroreference. After emptying the bladder, $50 \mathrm{ml}$ of warmed $\left(35-37^{\circ} \mathrm{C}\right)$ saline was instilled as a priming volume via the culture aspiration port. A clamp was applied to the tubing of the urinary drainage bag just distal to the culture aspiration port once the saline had filled the catheter and tubing up to that point. A pressure transducer was then inserted in the culture aspiration port via a 16 gauge needle and connected to the respiratory monitor (Dräger Cicero,
Dräger Medical, Lübeck, Germany), from which the IVP measurements were recorded. The system was calibrated with the patient in a supine position.

The pneumoperitoneum was created using insufflation of carbon dioxide. After proper positioning of the first trocar, the pneumoperitoneum was released to zero on the laparoscopy unit (Storz Endoscope, Karl Storz, Tuttlingen, Germany). This IAP measurement was used as the zero point, reflecting physiological IAP. IAP was then raised from 0 to $30 \mathrm{mmHg}$ using increments of $5 \mathrm{mmHg}$, during which the IVP was recorded end-expiratory after 5-10 $\mathrm{s}$ of equilibration.

After releasing the pneumoperitoneum, the tubing was held up against the measuring rod. Only then was the clamp removed, preventing drainage of priming volume into the bag. UC measurements were performed by repeating the steps as described above. The tubing was held up against the measuring rod and values recorded. The bladder was then emptied and instilled with $100 \mathrm{ml}$ of warmed saline, after which both IVP and UC measurements were repeated as described above.

Summarizing, we performed IVP and UC measurements with 50 and $100 \mathrm{ml}$ of saline as a priming volume, respectively, leading to four series of seven measurements per patient, using the laparoscopically measured IAP as standard.

All measurements were done by the same researcher (H. v d S.). For analysis, the unit for UC measurements was converted from centimeters of water $\left(\mathrm{cm} \mathrm{H}_{2} \mathrm{O}\right)$ to millimeters of mercury $(\mathrm{mmHg})(1 \mathrm{mmHg}=1.36 \mathrm{~cm}$ $\mathrm{H}_{2} \mathrm{O}$ ).

\section{Statistical analysis}

Correlation coefficients between IAP and both IVP and UC measurements with 50 and $100 \mathrm{ml}$ of saline were calculated with multiple regression analysis taking into account the within-patients variation. Bland and Altman plots were drawn. Bias values were calculated as the mean of difference between IVP and UC measurements, with its $95 \%$ limits of agreement (LA) for each volume (50 and $100 \mathrm{ml}$ ) using precision estimates (or SD of the bias). The percentage error was calculated as the LA divided by the mean IAP or mean IVP [13]. Partial correlation coefficients were estimated to control for the effect of BMI on the relation between IVP and UC measurements $(50$ and $100 \mathrm{ml})$. Statistical significance was accepted with a $p$ value less than 0.05 .

\section{Results}

The mean age of the 15 patients (10 females) was $45.1 \pm$ 11.2 years and the mean BMI was $27.3 \pm 4.3 \mathrm{~kg} / \mathrm{m}^{2}$. 
The IVP and UC measurements showed significant correlations with IAP using both priming volumes of $50 \mathrm{ml}$ ( $R=0.96$ and $0.92 ; p<0.001$, respectively) and $100 \mathrm{ml}$ of saline ( $R=0.93$ and $0.90 ; p<0.001$, respectively).

Comparing IVP and UC showed a correlation of 0.91 $(p<0.001)$ for a priming volume of $50 \mathrm{ml}$ and a correlation of $0.87(p<0.001)$ for $100 \mathrm{ml}$ of saline.

Correcting for the effect of BMI, partial correlations between IVP and UC were $0.91(p<0.001)$ for $50 \mathrm{ml}$ and $0.87(p<0.001)$ for $100 \mathrm{ml}$ of saline.

The differences between IVP and UC measurements as function of the mean of the IVP and UC measurements are presented in Bland and Altman plots, shown in Fig. 1. Using $50 \mathrm{ml}$ of saline, UC was $0.68 \mathrm{mmHg}$ higher than IVP with $95 \% \mathrm{LA}$ of -7.21 to $+5.85 \mathrm{mmHg}$. For $100 \mathrm{ml}$ of saline, UC was $1.23 \mathrm{mmHg}$ lower than IVP with $95 \%$ LA of -7.42 to $+9.87 \mathrm{mmHg}$ (Table 1 ).

\section{Discussion}

IAP has been shown to be a relevant parameter in critically ill patients. Measuring IVP using a pressure transducer is the most frequently described indirect method of estimating IAP. Our results show that both IVP and UC measurements reflect IAP. Moreover, the present study indicates that UC measurement could be a good clinical alternative to IVP measurement. Measuring UC instead of IVP has several advantages. It is simple, nontime consuming, easily reproducible and cheap.

Based on our experience and findings during this study, we are of the opinion that while performing a UC measurement, three criteria should be met. First, the meniscus of the UC must show respiratory variation. Secondly, the meniscus should remain stable in relation to the symphysis pubis, regardless of tube movement. Thirdly, applying gentle oscillations to the abdomen should be seen in the UC. These three conditions define a proper functioning UC. If these are not met, the most likely cause is the presence of an air bubble in the system.

In order to objectively appreciate the presented results, a few potential drawbacks of our study should be considered.

Using the symphysis pubis as a reference point has been common practice in performing IVP measurements. However, the exact localization of the symphysis pubis in daily practice is subject to interpretation due to differences in patient body habitus, and lack of consensus of using the bony rim or another point as the actual symphysis. Because of this variable interpretation, the midaxillary line at the level of the iliac crest, which is easier to palpate, even in obese patients, is now being advocated as the zero reference point $[6,14]$.

Our measurements were performed during valuable operating time. This allowed us only $5-10 \mathrm{~s}$ as
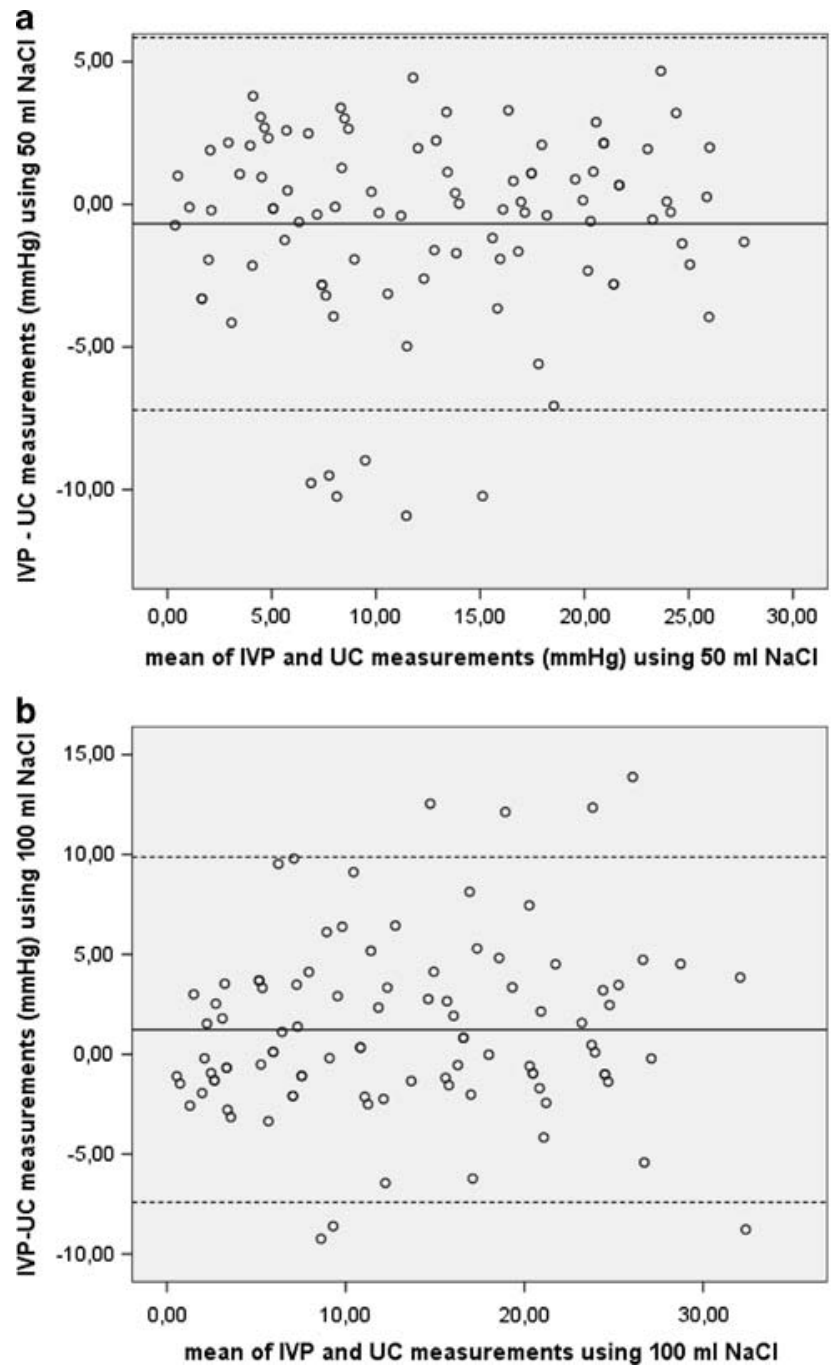

Fig. 1 a Bland and Altman plot displaying mean differences between IVP and UC measurements ( $\mathrm{mmHg}$ ) versus average of IVP and UC measurements $(\mathrm{mmHg})$, using $50 \mathrm{ml}$ of saline. The solid line represents the mean difference between both methods, and the dashed lines represent $95 \%$ limits of agreement. b Bland and Altman plot displaying mean differences between IVP and UC measurements $(\mathrm{mmHg})$ versus average of IVP and UC measurements $(\mathrm{mmHg})$, using $100 \mathrm{ml}$ of saline. The solid line represents the mean difference between both methods, and the dashed lines represent $95 \%$ limits of agreement

equilibration time. Chiumello et al. [15] performed a study using a priming volume of 50-200 ml, performing measurements after $5-10 \mathrm{~s}$, and after $5 \mathrm{~min}$ of equilibration. There was a significantly lower IAP at 5 min compared to 5-10 s, although only with $150-200 \mathrm{ml}$ as a priming volume. For 50 and $100 \mathrm{ml}$, the amounts generally used in clinical practice, this significance was not seen. Nevertheless, based on their findings, it was suggested that a sufficient equilibration time be used to allow for a reliable IVP measurement. Possibly in our study, using only 5-10 s of equilibration, IVP and UC may have been overestimated. 
Table 1 Results of Bland and Altman analysis comparing different techniques to measure intra-abdominal pressure

\begin{tabular}{lrlrrl}
\hline & \multicolumn{1}{c}{ Bias } & Precision & LLA & ULA & $\%$ Error $^{\mathrm{a}}$ \\
\hline IVP-UC 50 ml & -0.68 & 3.33 & -7.21 & 5.85 & 52.6 \\
IVP-UC 100 ml & 1.23 & 4.41 & -7.42 & 9.87 & 62.1 \\
IVP-IAP 50 ml & -2.50 & 3.25 & -8.95 & 4.00 & 43.2 \\
IVP-IAP 100 ml & -0.93 & 3.76 & -8.30 & 6.44 & 49.1 \\
IAP-UC 50 ml & 1.75 & 4.28 & -6.64 & 10.14 & 55.9 \\
IAP-UC 100 ml & 2.16 & 4.35 & -6.55 & 10.87 & 58.1 \\
\hline
\end{tabular}

a $\%$ Error calculated as the limits of agreement divided by the mean IAP $(15 \mathrm{mmHg})$ or mean IVP $(12.4 \mathrm{mmHg}$ for IVP $50 \mathrm{ml}$ and $13.9 \mathrm{mmHg}$ for IVP $100 \mathrm{ml}$ )

$I V P$ intra-vesicular pressure measurement, $U C$ urine column measurement, IAP intra-abdominal pressure, $L L A$ lower limit of agreement, ULA upper limit of agreement

The volume of bladder priming has been reported to act as a confounding factor $[16,17]$. For years priming volumes of 50-100 $\mathrm{ml}$ have been used $[8,15,18,19]$. Our present study protocol was based on these studies. Recently, however, a study was published in which priming with 50 and $100 \mathrm{ml}$ increased the measured IVP compared to $10 \mathrm{ml}$ [20]. Unfortunately, actual IAP was not measured and used as a reference.

Based on the available evidence, an international consensus group of critical care specialists stated that the reference standard for intermittent IAP measurement is via the bladder with a maximal instillation volume of $25 \mathrm{ml}$ of sterile saline [6].

Although BMI has been suggested to be a possible confounding factor [21], we did not find different correlations between IVP/UC and IAP when taking the BMI into account.

The Bland and Altman plots (Fig. 1) show that the difference between UC and IVP for both 50 and $100 \mathrm{ml}$ as a priming volume was lower than $1.3 \mathrm{mmHg}$. Recently, De Waele et al. [13] stated that in performing adequate IAP measurements, bias should be $0 \mathrm{mmHg}$ (range -1 to $+1 \mathrm{mmHg}$ ) with maximum allowed LA within a range of $4 \mathrm{mmHg}$ ( $\mathrm{LA}=$ bias $\pm 4 \mathrm{mmHg}$ ). Yet, due to our small study population, the LA were rather wide (up to $-8.95 \mathrm{mmHg}$ and $+10.85 \mathrm{mmHg}$ ).
The plots also show that the distribution does not change with increasing pressure. The results suggest that UC measurements apply even at higher pressure ranges, although a larger study population is needed to substantiate this.

The manner in which we performed our measurements in the present surgical setting might have been of influence on the obtained results. Using the sequence of IVP and UC measurement at 50 and $100 \mathrm{ml}$, respectively, in three patients we observed that after releasing an IAP of $30 \mathrm{mmHg}$ (finishing the IVP measurement), the zero measurement of the following UC was surprisingly high compared to the previously measured zero pressure of IVP. We hypothesize that due to intrinsic detrusor activity the bladder does not have the ability to relax fast enough before proceeding to next measurements. As this study was performed during valuable operating time, we were unable to prove this hypothesis. Nevertheless, we found a significant correlation between IVP and UC measurements. In order to limit any possible intrinsic detrusor activity due to sudden temperature changes, the priming fluid was warmed to body temperature $\left(35-37^{\circ} \mathrm{C}\right)$ before instilling.

This study was conducted in a surgical setting, with patients relatively healthy and under general anesthesia. Whether our findings can be extrapolated to the critical care patient with signs of the ACS is subject to further investigation.

\section{Conclusion}

Based on our results we conclude that UC measurement could be a good, non-invasive clinical alternative to IVP measurement as an estimation of the IAP.

Acknowledgment We wish to thank Dr. Yvonne Hoogeveen for revising the paper.

Open Access This article is distributed under the terms of the Creative Commons Attribution Noncommercial License which permits any noncommercial use, distribution, and reproduction in any medium, provided the original author(s) and source are credited.

\section{References}

1. Malbrain ML, Chiumello D, Pelosi P, Wilmer A, Brienza N, Malcangi V, Bihari D, Innes R, Cohen J, Singer P, Japiassu A, Kurtop E, De Keulenaer BL, Daelemans R, Del Turco M, Cosimini P, Ranieri M, Jacquet L, Laterre PF, Gattinoni L (2004) Prevalence of intra-abdominal hypertension in critically ill patients: a multicentre epidemiological study. Intensive Care Med 30:822-829
2. Cheatham ML, Malbrain ML, Kirkpatrick A, Sugrue M, Parr M, De Waele J, Balogh Z, Leppäniemi A, Olvera C, Ivatury R, D'Amours S, Wendon J, Hillman K, Wilmer A (2007) Results from the International Conference of Experts on Intraabdominal Hypertension and Abdominal Compartment Syndrome. II. Recommendations. Intensive Care Med 33:951-962
3. Malbrain ML, De Laet I, Cheatham ML (2007) Consensus conference definitions and recommendations on intra-abdominal hypertension (IAH) and the abdominal compartment syndrome (ACS) - the long road to the final publications, how did we get there? Acta Clin Belg Suppl 62:44-59 
4. Cheatham ML, White MW, Sagraves SG, Johnson JL, Block EF (2000) Abdominal perfusion pressure: a superior parameter in the assessment of intra-abdominal hypertension. J Trauma 49:621-627

5. Ivy ME, Atweh NA, Palmer J, Possenti PP, Pineau M, D'Aiuto M (2000) Intraabdominal hypertension and abdominal compartment syndrome in burn patients. J Trauma 49:387-391

6. Malbrain ML, Cheatham ML, Kirkpatrick A, Sugrue M, Parr M, De Waele J, Balogh Z, Leppäniemi A, Olvera C, Ivatury R, D'Amours S, Wendon J, Hillman K, Johansson K, Kolkman K, Wilmer A (2006) Results from the International Conference of Experts on Intra-abdominal Hypertension and Abdominal Compartment Syndrome. I. Definitions. Intensive Care Med 32:1722-1732

7. Malbrain ML (2004) Different techniques to measure intra-abdominal pressure (IAP): time for a critical reappraisal. Intensive Care Med 30:357371

8. Kron IL, Harman PK, Nolan SP (1984) The measurement of intra-abdominal pressure as a criterion for abdominal reexploration. Ann Surg 199:28-30

9. Iberti TJ, Lieber CE, Benjamin E (1989) Determination of intraabdominal pressure using a transurethral bladder catheter: clinical validation of the technique. Anesthesiology 70:47-50
10. Cheatham ML, Safcsak K (1998) Intraabdominal pressure: a revised method for measurement. J Am Coll Surg 186:594-595

11. Harrahill M (1998) Intra-abdominal pressure monitoring. J Emerg Nurse 24:465-466

12. van der Steeg HJJ, Sewing ACP, van Akkeren JP, Roumen RMH (2007) Urine column measurement as an estimation of intra-abdominal pressure: comparison with intra-vesicular pressure measurement as the 'gold standard'. Acta Clin Belg Suppl 62:285

13. De Waele JJ, De Laet I, Malbrain ML (2007) Rational intraabdominal pressure monitoring: how to do it? Acta Clin Belg suppl 62:16-25

14. De Waele JJ, De Laet I, De Keulenaer B, Widder S, Kirkpatrick AW, Cresswell AB, Malbrain M, Bodnar Z, Mejia-Mantilla JH, Reis R, Parr M, Schulze R, Compano S, Cheatham M (2008) The effect of different reference transducer positions on intra-abdominal pressure measurement: a multicenter analysis. Intensive Care Med 34:1299_ 1303

15. Chiumello D, Tallarini F, Chierichetti M, Polli F, Li Bassi G, Motta G, Azzari S, Carsenzola C, Gattinoni L (2007) The effect of different volumes and temperatures of saline on the bladder pressure measurement in critically ill patients. Crit Care 11:R82
16. Verbrugghe W, Van Mieghem N, Daelemans R, Lins R, Malbrain ML (2003) Estimating the optimal bladder volume for intra-abdominal pressure measurement by bladder pressurevolume curves. Crit Care Suppl 7:184

17. Malbrain ML, Deeren DH (2006) Effect of bladder volume on measured intravesicular pressure: a prospective cohort study. Crit Care 10:R98

18. Fusco MA, Martin RS, Chang MC (2001) Estimation of intra-abdominal pressure by bladder pressure measurement: validity and methodology. J Trauma 50:297-302

19. Johna S, Taylor E, Brown C, Zimmerman G (1999) Abdominal compartment syndrome: does intracystic pressure actual reflect intraabdominal pressure? A prospective study in surgical patients. Crit Care 3:135-138

20. De Waele J, Pletinckx P, Blot S, Hoste E (2006) Saline volume intransvesical intra-abdominal pressure measurement: enough is enough. Intensive Care Med $32: 455-459$

21. Luca A, Cirera I, Garcia-Pagan JC, Feu F, Pizcueta P, Bosch J, Rodes J (1993) Hemodynamic effects of acute changes in intra-abdominal pressure in patients with cirrhosis. Gastroenterology 104:222-227 